Justyna Groblińska, Katarzyna Kowalik

\title{
GLI SLAVI DAL PUNTO DI VISTA ITALIANO - DIFFICOLTÀ TRADUTTOLOGICHE NELLA TRADUZIONE DE GLI SLAVI. STORIA, CULTURA E LINGUE DALLE ORIGINI AI NOSTRI GIORNI DI MARCELLO GARZANITI
}

\author{
Slavs from the Italian perspective: difficulties in the translation \\ of Gli slavi. Storia, cultura e lingue dalle origini ai nostri giorni by \\ Marcello Garzaniti
}

\begin{abstract}
The article is an effect of cooperation of the authors in the translation of the Marcello Garzaniti's monograph Gli slavi. Storia, cultura e lingue dalle origini ai nostri giorni from Italian into Polish. The translators classify, describe and analyze causes and effects of such phenomena as: different vocabulary of two languages, syntax transfer from Italian to Polish, lexical and stylistic unification of text elaborated by three different translators, material errors appearing in the source text, cases of obsolescence in the original version, scientific style, author's idiolectal expressions, development of knowledge in the translation process, Polish culture's perception in Italy and its influence on translator's decisions. The discussed issues represent a synthesis of problems with which a translator from Italian into Polish can face.
\end{abstract}

Key words: cultural differences in translation, scientific translation, specialised translation, translation errors, stages of translator's work, written translation

Parole chiave: differenze culturali nella traduzione, traduzione scientifica, traduzione specializzata, errori nella traduzione, fasi del lavoro del traduttore, traduzioni scritte

Słowa kluczowe: różnice kulturowe w przekładzie, tłumaczenie naukowe, tłumaczenie specjalistyczne, błędy tłumaczeniowe, etapy pracy tłumacza, tłumaczenie pisemne

\section{Introduzione}

La monografia Gli slavi. Storia, cultura e lingue dalle origini ai nostri giorni di Marcello Garzaniti è stata pubblicata nel 2013 dalle edizioni Carocci a Roma. L'argomento slavo e la vasta elaborazione sono stati probabilmente i motivi per i quali le edizioni polacche Wydawnictwo Uniwersytetu Łódzkiego hanno scelto di 
pubblicare un libro scientifico riguardante il mondo slavo dal punto di vista italiano. Pertanto, tre traduttrici (di cui due figurano come autrici di questo articolo) sono state invitate al progetto traduttologico che riguardava la traduzione del libro dall'italiano in polacco. Sono stati impiegati nove mesi per la fase di traduzione dell'intero testo (467 pagine), che è stato diviso in tre parti uguali. Ogni traduttrice ha dovuto dunque tradurre 156 pagine di un testo scientifico notevolmente variegato. Le difficoltà evidenti, già segnalate nel titolo, riguardano la ricchezza del testo che esige dal traduttore una conoscenza molto vasta delle discipline: linguistica, architettura, storia dell'arte, filosofia, teologia, storia universale e altre. La scelta di dividere un materiale così ampio e difficile fra tre persone ha posto di conseguenza degli ostacoli all'unificazione stilistica finale. Nei paragrafi seguenti verranno esposte le caratteristiche dei problemi emersi nel corso della traduzione scientifica. Nonostante la particolarità della traduzione del testo scientifico, le fasi seguenti rispondono al classico schema del lavoro traduttologico che descrive H. Dzierżanowska ${ }^{1}$ 1) analisi, 2) traduzione, 3) correzione, 4) redazione stilistica. La complessità del testo originale esigeva anzi l'amplificazione di alcuni processi, perciò in questo caso enumereremo le tappe come segue:

1) analisi;

2) traduzione;

3) verifica della traduttrice del frammento;

4) verifica della seconda traduttrice;

5) verifica della terza traduttrice;

6) correzione;

7) redazione stilistica (naturalmente le tappe quarta e quinta hanno il carattere reciproco e valgono per ogni parte del testo tradotto).

Focalizzandosi sulla parte di traduzione e dei problemi ad essa connessi, il presente articolo non include le tappe della verifica dei redattori scientifici che seguono la traduzione generale e costituiscono una parte molto importante della redazione.

\section{Caratteristiche della traduzione scientifica}

Analizzando il tema molto complesso della traduzione scientifica non è possibile ignorare la constatazione espressa da Elżbieta Skibińska: "[s]econdo la convinzione generale (stereotipica, idealistica) la scienza, che costituisce il bene comune dell'umanità, ha un carattere soprannazionale e sopralinguistico e l'accesso a essa è aperto a tutti grazie alla circolazione

${ }^{1}$ Cfr. H. Dzierżanowska, Tłumaczenie tekstów nieliterackich. Założenia teoretyczne i wskazówki metodyczne, Wydawnictwa Uniwersytetu Warszawskiego, Warszawa 1977, p. 77-89. 
internazionale del pensiero" ${ }^{2}$. Sembrerebbe quindi che la traduzione dei testi scientifici, argomento di enorme importanza, fosse frequentemente analizzato nelle ricerche degli specialisti di traduttologia. Infatti, se incorporiamo il problema della traduzione di questo tipo di testi nel contesto della traduzione specializzata - medica, economica, giuridica, tecnica - possiamo trovare numerose pubblicazioni dedicate a questo campo ${ }^{3}$. Soltanto negli ultimi anni la questione è stata considerata nei lavori dei teorici di traduzione come Krzysztof Hejwowski ${ }^{4}$, Jerzy Pieńkos ${ }^{5}$, Alicja Pisarska e Teresa Tomaszkiewicz ${ }^{6}$.

Tuttavia, in corso d'opera le traduttrici hanno notato che nelle opere critiche ci si concentra più raramente sulla traduzione dei testi umanistici, per esempio quelli dedicati alla linguistica, letteratura, storia, anche se essi senza alcun dubbio appartengono al vasto ambito dei testi specialistici. Kopczyńska distingue le seguenti caratteristiche del linguaggio specialistico nei testi storici che riguardano allo stesso modo altre discipline umanistiche: i testi contengono una specifica terminologia, hanno un caratteristico stile e registro, si riferiscono a una certa realtà culturale, sono indirizzati a lettori ben precisi e vengono pubblicati con uno scopo concreto. Comunque, si deve osservare che i loro elementi indispensabili, come le citazioni, la bibliografia e le note li differenziano dagli altri tipi di traduzione ${ }^{7}$. L'autonomia e la particolarità dei testi scientifici costituiscono due motivi principali per i quali la loro traduzione esige degli studi più approfonditi. Attualmente la fonte più esauriente delle informazioni teoriche e pratiche riguardanti la traduzione scientifica, in questo caso soprattutto quella legata alla linguistica, è rappresentata dalla raccolta di Zofia Kozłowska $O$ przekładzie tekstu naukowego (na materiale tekstów językoznawczych) ${ }^{8}$ e la monografia a cura di A. Duszak, A. Jopek-Bosiacka e G. Kowalski Tekst naukowy i jego przekład’.

2 E. Skibińska, Miejsce przekładów w „Tekstach” i „Tekstach Drugich”, [in:] Tekst naukowy i jego przektad, a cura di A. Duszak, A. Jopek-Bosiacka, G. Kowalski, Universitas, Kraków 2015, p. 145.

${ }^{3}$ M. Kopczyńska, Przekład tekstów specjalistycznych - oczekiwania i wyzwania a praktyka, "Rocznik Przekładoznawczy. Studia nad teorią, praktyką i dydaktyką przekładu" 2015, v. 10, p. 113-126, http://dx.doi.org/10.12775/RP.2015.006 (accesso: 29.12.2017).

${ }^{4} \mathrm{~K}$. Hejwowski, Kognitywno-komunikacyjna teoria przekładu, Wydawnictwo Naukowe PWN, Warszawa 2006.

${ }^{5}$ J. Pieńkos, Podstawy przekładoznawstwa. Od teorii do praktyki, Wydawnictwo Zakamycze, Kraków 2003.

${ }^{6}$ A. Pisarska, T. Tomaszkiewicz, Wspótczesne tendencje przekładoznawcze, Wydawnictwo Naukowe UAM, Poznań 1996.

7 M. Kopczyńska, Przekład tekstów specjalistycznych ..., p. 115.

8 Z. Kozłowska, O przekładzie tekstu naukowego (na materiale tekstów jezzkoznawczych), Wydawnictwa Uniwersytetu Warszawskiego, Warszawa 2007.

9 Tekst naukowy i jego przekład, a cura di A. Duszak, A. Jopek-Bosiacka, G. Kowalski, Universitas, Kraków 2015. 
Con la speranza che i volumi menzionati siano un segno d'interesse sempre crescente verso la problematica che affrontiamo, crediamo che le ricerche sulla traduzione scientifica debbano essere continuate, soprattutto perché condividiamo pienamente la convinzione di Anna Duszak secondo la quale "la comunità accademica del discorso è inseparabilmente legata all'idea dell' 'identità accademica"10.

\section{Metodologia}

L'obiettivo dell'articolo si racchiude nella tipologia e nella descrizione dei problemi tipici che emergono nella traduzione del testo scritto scientifico (come ad esempio il modo di trasmettere le informazioni, la terminologia adatta, la trasmissione del punto di vista dell'autore, ecc. $)^{11}$. Le nostre osservazioni e conclusioni si basano soprattutto sull'esperienza pratica della traduzione del libro di Marcello Garzaniti. Avendo chiuso il processo della traduzione, il materiale composto dal testo originale, dai prototipi e dalle ultime versioni del testo finale è stato sottoposto alla verifica teorica. Le traduttrici hanno analizzato, classificato ed elaborato tipiche difficoltà traduttologiche che avevano affrontato. L'analisi dei commenti, dubbi e varie fonti aggiuntive hanno permesso di evidenziare le tendenze delle difficoltà. Nonostante le diverse specializzazioni delle traduttrici (linguistica, letteraria, culturale), i loro problemi sono stati prevalentemente simili, perciò si può concludere il loro carattere universale. Le sfide si dividono in sei categorie delle difficoltà: universali, stilistiche, riguardanti il sistema linguistico, culturali, riguardanti disavanzi nel processo traduttologico e quelle riguardanti errori e obsolescenze.

\section{Difficoltà}

Le difficoltà universali emergevano dalla prima tappa (l'analisi) del testo tradotto, cominciando dalla complessità generale del libro e finendo con la percezione del mondo slavo dalle traduttrici slave attraverso il punto di vista italiano dell'autore che a volte si avvicinava, ma anche spesso si allontanava dalla veloce e chiara comprensione del concetto. Le sfide stilistiche riguardavano

10 A. Duszak, Wielogłosowość języków nauki a tożsamość akademicka w świetle lingwistyki stosowanej, [in:] Tekst naukowy i jego przekład, p. 81.

11 Cfr. M. Kopczyńska, Przekład tekstów specjalistycznych..., p. 114-115; M. Kopczyńska, cit. da: Z. Kozłowska, O przekładzie tekstu naukowego..., p. 55-56. 
ovviamente il mantenimento dello stile scientifico generale ${ }^{12}$ (terminologia precisa, obiettività, impersonalità ${ }^{13}$ e simili), ma anche la riflessione dell'idioletto dell'autore ${ }^{14}$ e l'unificazione di tre stili diversi delle traduttrici (soprattutto nell'aspetto terminologico, ma anche legato alla denominazione). Le differenze tra i sistemi linguistici si segnalavano piuttosto nella sintassi (soprammenzionata) e nella corcondanza dei tempi (nel testo originale a volte viene usato il tempo presente per descrivere le azioni passate, ciò anche in polacco è possibile, con la coerenza dell'uso). Le sfumature culturali similmente a quelle universali si espongono fin dall'inizio della traduzione. Già le origini diverse dell'autore e delle traduttrici creano la difficoltà alla base della traduzione stessa. In questo caso però, il confine culturale è stato raddoppiato, visto che l'autore rappresenta in questo testo la realtà delle traduttrici e dei futuri lettori. Tale situazione meno tipica è da un lato favorevole per le traduttrici, ma dall'altro lato impone la doppia responsabilità del traduttore che in questo caso dovrebbe essere un naturale esperto. In realtà, a volte la relazione delle traduttrici con il testo funzionava in questo modo ed è stata vantaggiosa per loro (la possibilità della valutazione critica e della sensibilità che permette di trovare degli errori possibili), a volte invece si è rivelata esigente e ha richiesto alle traduttrici un livello molto alto di conoscenza dettagliata di argomenti specialistici (ad esempio la fonetica dello slavo ecclesiastico). In alcuni casi l'autore si riferiva alle fonti non tradotte nella lingua polacca, il che provocava una verifica meno precisa di un'informazione. Alla fine, sono emersi alcuni errori dell'autore che richiedevano una doppia o tripla verifica, visto che il traduttore del testo scientifico dovrebbe avere fiducia nell'autore esperto, ma ovviamente tale situazione può capitare e scarsamente appare anche nei testi scientifici. Alcuni invece sono naturali: si legano strettamente con il passare del tempo e il fenomeno dell'obsolescenza.

12 Cfr. A. Wilkoń, Typologia odmian językowych współczesnej polszczyzny, Wydawnictwo Uniwersytetu Śląskiego, Katowice 2000, p. 63-67 (in particolare p. 65).

13 Le forme impersonali prevalgono nei testi scientifici polacchi, mentre nei testi paralleli italiani più spesso si trovano le forme personali, particolarmente nella prima persona plurale - cfr. M. Garzaniti, Gli slavi. Storia, cultura e lingue dalle origini ai nostri giorni, Carocci editore, Roma 2013 , p. 15, 17, 20 e altre; i pronomi possessivi della prima persona plural - ibidem, p. 23 e altre. Si capisce che l'intenzione dell'autore italiano non è l'ignoranza dello stile scientifico, ma sicuramente l'intenzione di sottolineare il carattere collettivo della monografia.

14 A volte la riduzione delle ripetizioni (specialmente quelle sintattiche) che nella lingua polacca si potevano sostituire con il valore aggiuntivo per il testo finale (già la terminologia scientifica esige che gli autori usino il linguaggio preciso e ripetitivo, perciò la sintassi dovrebbe essere più diversificata, se possibile). 


\subsection{Difficoltà universali}

La sfida principale nella traduzione del testo consisteva soprattutto nella sua ampiezza ed eterogeneità. L'opera di Marcello Garzaniti è un complesso manuale di storia, lingue, cultura e politica dei popoli slavi nel corso di tre millenni. Inoltre, al di là della problematica della Slavia, l'autore presenta anche un ampio contesto europeo e mondiale che avvicina e spiega gli avvenimenti storici e culturali di quella parte d'Europa poco conosciuta agli italiani. Questa soluzione sembra indispensabile per i lettori italiani dato che la monografia mostra dettagliatamente numerosi e molto complicati aspetti della realtà slava: l'economia, l'arte, la letteratura, il folclore, la religione, la mitologia, la filosofia, la legge, l'agricoltura, l'archeologia e tanti altri. Il lavoro delle traduttrici esigeva quindi un'adeguata preparazione, le consultazioni con specialisti nel quadro di queste discipline e, in generale, un' immersione totale nella problematica dell'opera. Pertanto, quello che ha confermato il progetto traduttivo descritto, è la necessità di uno sviluppo continuo nella professione del traduttore - gli esempi tratti dell'esperienza menzionata mostrano che senza le letture e le ricerche facoltative non sarebbe stato possibile superare diverse difficoltà.

\subsection{Difficoltà stilistiche}

Lo stile dell'autore appare pure nel testo di carattere scientifico, anche se è uno dei registri più oggettivi e impersonali. Tre traduttrici hanno notato le costruzioni e i termini tipici per M. Garzaniti. Lautore usa molto spesso il nesso correlativo non solo... ma per dimostrare quanto spesso si incontrano le formule ripetitive, si presenta un frammento del testo con evidenziate in grassetto le costruzioni tipiche:

La Russia da secoli non solo convive con le popolazioni finniche, ma espandendosi nell'area asiatica ha colonizzato ampi territori abitati dalle popolazioni turco - mongole fino a raggiungere la Siberia e l'Alaska. Il collasso dell'Unione Sovietica, erede dell'impero russo, ha provocato non solo una profonda crisi interna, ma ha determinato la nascita di una serie di minoranze russe all'estero, sia nei paesi baltici sia nell'Asia centrale. Sul mar Baltico la Russia non solo ha costruito la sua antica capitale San Pietroburgo, già Leningrado, ma possiede anche il territorio della Prussia orientale con la città Kaliningrad, la vecchia Konigsberg, separata dal resto del paese e incuneata all'interno dell'Unione europea ${ }^{15}$.

La citazione del frammento ha lo scopo di illustrare il fenomeno e di sottolineare la tendenza che riguarda quasi ogni autore, anche scientifico.

15 M. Garzaniti, Gli slavi..., p. 30-31. 
Le traduttrici hanno deciso di ricostruire a volte alcune delle frasi ripetute ciò corrisponde con l'idea di $\mathrm{H}$. Dzierżanowska che sottolinea la priorità del messaggio rispetto alla mantenimento delle particolarità dello stile di autore ${ }^{16}$ (del suo idioletto).

Lo stile dell'autore del testo originale viene confrontato con lo stile del traduttore, cioè il coautore della versione tradotta. In questo caso, la traduzione effettuata da tre traduttrici ha causato l'esigenza dell'unificazione del testo tradotto dal punto di vista linguistico, stilistico e generale. È impossibile tradurre un testo allo stesso modo ${ }^{17}$, quindi ancora più difficile sarebbe stato tradurre tre parti del libro secondo una chiave stilistica. Le differenze delineatesi subito dopo la prima verifica, erano quelle lessicali. Ce ne sono diversi esempi. L'aggettivo fondamentale a volte veniva tradotto come fundamentalny (il lemma più vicino dal punto di vista etimologico e formale, bensì in alcuni casi funziona nel testo polacco come il calco linguistico e non l'equivalente più adatto) oppure come kluczowy. La scelta definitiva dipendeva dal contesto della frase che comprendeva l'aggettivo. Laltro caso non riguarda la difficoltà del trovare il lemma, ma la sua versione ortografica polacca. L'aggettivo bizantino in polacco si traduce come bizantyjski oppure bizantyński. Ambedue le scritture sono corrette ${ }^{18}$, delle quali una deriva dal nome della nazionalità Bizantyjczyk, l'altra rispettivamente - Bizantyńczyk. La scelta tra le versioni ortografiche aveva piuttosto il carattere preferenziale e usuale. La discrepanza che esigeva l'unificazione senza eccezioni riguardava il toponimo storico Porta sublime. Gli equivalenti polacchi erano tre: Wysoka Porta, Porta Otomańska, Porta Osmańska. Per la frequenza più alta dell'uso del primo nome Wysoka Porta le traduttrici hanno deciso di scegliere quest'onimo. Fatta eccezione per il lessico, si elaborava il testo nell'aspetto dello stile di ogni traduttrice e si cercava di ridurre le sfumature fra tre approcci diversi, i quali talvolta si avvicinavano alle costruzioni sintattiche italiane, in altri casi venivano meglio trasformate nelle costruzioni tipiche polacche.

Il confronto del traduttore con lo stile è indispensabile per ogni traduzione. In questo caso le traduttrici si sono attenute in maniera stretta e precisa allo stile scientifico. Hanno dovuto dunque trasporre purezza e trasparenza del comunicato. Per di più, a volte hanno dovuto semplificare le frasi italiane che tendono quasi sempre ad essere più complesse da quelle polacche, ciò nel caso del testo finale scientifico polacco è ancora più importante. Questo aspetto collega le difficoltà stilistiche con quelle sintattiche.

${ }_{16}$ Cfr. H. Dzierżanowska, Tłumaczenie tekstów nieliterackich..., p. 6.

${ }_{17}$ Cfr. M. Kopczyńska, Przekład tekstów specjalistycznych ..., p. 117; K. Hejwowski, Kognitywno-komunikacyjna teoria przekładu..., p. 125.

${ }_{18}$ Cfr. Wielki stownik poprawnej polszczyzny PWN, a cura di A. Markowski, Wydawnictwo Naukowe PWN, Warszawa 2004, p. 76. 


\subsection{Difficoltà riguardanti il sistema linguistico}

Oltre alle sopramenzionate questioni di merito, durante il lavoro traduttivo sono apparse anche altre sfide risultanti direttamente dalle caratteristiche dei testi scientifici italiani. Le traduttrici dovevano scegliere molte volte tra la fedeltà al testo originale e la necessità di adattarlo alle regole del sistema linguistico della lingua polacca e alla tradizione dello stile accademico nel nostro paese. Molto spesso nella traduzione si dovevano introdurre dei cambiamenti nell'uso dei tempi verbali. Marcello Garzaniti, avendo probabilmente l'intenzione di rendere il testo più vivace e dinamico, usava frequentemente il tempo presente storico, come nell'esempio: "Per la prima volta compaiono sulle carte europee i nuovi Stati indipendenti dell'Ucraina e della Bielorussia, ai quali si aggiungono la Macedonia, designata ufficialmente con l'acronimo FYROM (Former Yugoslav Republic of Macedonia, cfr. riquadro 4, p. 27), e il piccolo Montenegro"19. Analizzando i frammenti particolari con l'uso di questa soluzione, è stato deciso nella maggior parte dei casi di applicare invece gli adeguati tempi passati che hanno reso il testo polacco di carattere storico più coerente.

Nonostante lo stile scientifico polacco si caratterizzi per la complessità della sua sintassi, l'opera Gli Slavi ha affermato la convinzione che le abitudini retoriche italiane superano ancora questa tendenza. La versione originale del testo più volte chiede da parte del lettore uno sforzo di concentrazione sulle strutture costruite dalle frasi estremamente complesse, come quella riportata sotto:

Appare dunque lodevole lo sforzo di aprire la ricerca alle «aree solitamente ritenute marginali rispetto al contesto europeo», ma allo stesso tempo questo tentativo appare limitato a registrare la realtà medievale «delle molte Europe possibili», cioè «la prospettiva di un'Europa bizantina o islamica (ma anche magiara, o slava, o vichinga)», senza impegnarsi in uno sforzo ermeneutico delle dinamiche storiche che non sia un generico «riferimento ai processi di europeizzazione (o forse soltanto di raccordo) delle immense regioni confinarie (slave e baltiche anzitutto) $\gg^{20}$.

Secondo le traduttrici non è stato possibile mantenere lo stesso schema delle frasi. Analogamente ai numerosi esempi delle difficoltà descritte nel presente articolo, nel caso di dubbi concernenti le differenze tra due sistemi linguistici, prevaleva l'idea di proporre ai lettori la versione conforme alle abitudini stilistiche polacche. Il metodo appare adeguato alla luce delle parole di Krzysztof Hejowski che dichiara che "il fatto di non prendere in considerazione la situazione del destinatario può decidere dell'inutilità totale della traduzione”21.

19 M. Garzaniti, Gli slavi..., p. 23.

20 Ibidem, p. 17.

${ }^{21}$ K. Hejwowski, Kognitywno-komunikacyjna teoria przekładu..., p. 138, "niebranie pod uwagę sytuacji odbiorcy może zadecydować o całkowitej nieprzydatności przekładu”. 


\subsection{Difficoltà culturali}

La differenza culturale costituisce l'aspetto essenziale della traduzione in generale ${ }^{22}$ : essa è legata strettamente al confronto delle due lingue. Nella situazione tipica e più frequente il traduttore deve presentare un alto interesse verso la cultura dell'autore che viene esposta nel testo ${ }^{23}$. In questo caso particolare le traduttrici rappresentano la cultura descritta slava, ma il loro compito era tuttora più complesso ${ }^{24}$, dato che si doveva capire l'approccio italiano al mondo slavo. La prima questione riguarda l'esplicitazione superflua del testo italiano dal punto di vista del futuro lettore polacco ${ }^{25}$, specialmente se fosse un esperto (la situazione molto probabile per il motivo del carattere scientifico del testo). Come l'illustrazione del fenomeno si può presentare Casimiro il Grande dalla Polonia ${ }^{26}$ che ovviamente è tradotto come Kazimierz Wielki senza la sua provenienza che per il lettore polacco è ridondante. L'altro aspetto delle differenze tra il testo originale e il testo tradotto è posto in altri casi dai nomi personali ${ }^{27}$, ad esempio gli italiani tendono a presentare i personaggi russi in modo seguente: Aleksandr Sergeevič Puškin, mentre i polacchi omettono il patronimico e conoscono il poeta russo come Aleksander Puszkin. Per di più, il testo italiano usa spesso la denominazione tedesca di alcuni toponimi ${ }^{28}$ - ad esempio la battaglia di Tannenberg - bitwa pod Grunwaldem. In Polonia la denominazione Tannenberg non sarà riconosciuta. Risulta che la diversità culturale si riflette prima di tutto al livello onomastico. Per mostrare quanto evidente è l'approccio italiano ci serviranno due esempi. In primo luogo il nome della città croata Dubrovnik non è stato adottato e italianizzato, lo si sostituisce con il nome uguale a quello

22 L'argomento discusso ed elaborato dai diversi punti di vista in due ampi capitoli - cfr. K. Hejwowski, Kognitywno-komunikacyjna teoria przekładu..., p. 48-104.

23 È una delle condizioni che formula H. Dzierżanowska per i traduttori - cfr. H. Dzierżanowska, Tłumaczenie tekstów nieliterackich..., p. 12.

24 Secondo O. Wojtasiewicz le differenze culturali possono creare più difficoltà nella traduzione che il livello lessicale apparentemente più soffisticato - cfr. O. Wojtasiewicz, Wstęp do teorii tłumaczenia, Wydawnictwo TEPIS, Warszawa 1996, p. 82-83.

25 Sull'importanza del futuro lettore - cfr. K. Hejwowski, Kognitywno-komunikacyjna teoria przekładu..., p. 138.

${ }^{26}$ M. Garzaniti, Gli slavi..., p. 276.

27 Simili problemi segnala M. Kopczyńska - cfr. M. Kopczyńska, Przekład tekstów specjalistycznych..., p. 121 e K. Hejwowski - cfr. K. Hejwowski, Kognitywno-komunikacyjna teoria przektadu..., p. 69.

28 Anche la problematica dei toponimi viene descritta da M. Kopczyńska che propone le risoluzioni più esplicite, però nel suo caso la direzione della traduzione e contraria (dal polacco in inglese) e l’argomento riguarda la Prussia - cfr. M. Kopczyńska, Przekład tekstów specjalistycznych..., p. 120-121. 
del comune siciliano - Ragusa ${ }^{29}$. Per il lettore polacco il toponimo Ragusa fa riferimento alla geografia italiana, non croata, quindi indubbiamente in questo caso l'equivalente è il toponimo Dubrownik. L'esempio che conclude questo discorso e sottolinea le caratteristiche essenziali riguarda la percezione di Praga: "La città di Praga, sede della corte imperiale e reale, pur non potendosi confrontare con le maggiori città italiane per ricchezza e grandezza, era in una fase di grande sviluppo e diventò una delle città più importanti dell'Europa centrale" 30 . La frase citata dimostra quanto forte è l'identità culturale italiana e la convinzione che le città in altri Paesi, anche se in pieno sviluppo, non possono competere con qualsiasi città italiana. Non negando la grandezza dell'architettura italiana, si nota la soggettività del frammento che contrappone al carattere obiettivo del testo intero.

Le differenze culturali emergevano ancora significativamente nelle formule legate alla soggettività: "A. Mickiewicz (1798-1855) e A. S. Puškin (17991837), entrambi sudditi dello zar russo"31, "Jan Kochanowski, il maggior poeta polacco" 32 . In Polonia nessuno direbbe che Mickiewicz fosse stato un suddito dello zar russo - i polacchi non si sentirono mai sottomessi allo zar anche se Polonia in quel periodo non fu libera. Per quanto riguarda la valutazione di Kochanowski come scrittore, fu il maggior poeta rinascimentale e senza dubbio uno dei maggiori poeti in tutta la storia, ma sono le formule interamente soggettive e sarebbero controverse se fossero state tradotte letteralmente nella lingua polacca.

\subsection{Difficoltà riguardanti disavanzi nel processo traduttologico}

L'eterogeneità della monografia di Garzaniti si manifestava non solo nella molteplicità degli argomenti trattati, ma anche nell'uso frequente delle fonti letterarie e storiche. Lautore ha arricchito il volume con numerose citazioni dalle cronache, dei brani di letteratura oppure con dei frammenti dei documenti ${ }^{33}$. Ovviamente, le traduttrici sono partite dalla premessa secondo la quale prima di tutto si è obbligati a trovare le versioni dei testi che funzionano già nella lingua d'arrivo. Le traduzioni proprie sono accessibili, ma in tale caso occorre giustificare il motivo per il quale si ha bisogno di una nuova versione, e non quella che esiste nell'immaginazione comune dei destinatari ${ }^{34}$.

29 Cfr. M. Garzaniti, Gli slavi... - ad esempio p. 292, 293, 295 e altre.

30 Ibidem, p. 281.

31 Ibidem, p. 364.

32 Ibidem, p. 300.

33 M. Kopczyńska, Przekład tekstów specjalistycznych ..., p. 115.

34 Ibidem. 
Durante il lavoro è stato scoperto che un grande numero dei testi non era mai stato pubblicato in Polonia. Per questo motivo le traduttrici hanno dovuto affrontare il problema dello stile nella traduzione. Il linguaggio scientifico del testo principale veniva intrecciato dalle cronache medievali, dalla poesia popolare e dai testi monastici. Si deve aggiungere inoltre che alcune opere citate non hanno nemmeno la versione polacca del titolo, come Il Canto di Ostrov (nella traduzione presentata Pieśń Ostrova) oppure Il Racconto di Boris e Gleb (Opowieść Borysa i Gleba). In tali situazioni gli elementi mancanti dovevano essere tradotte in maniera filologica dalle autrici della versione polacca.

\subsection{Difficoltà riguardanti errori e obsolescenze}

Avendo sempre in memoria il fatto che la monografia avrebbe svolto il ruolo del manuale accademico, le traduttrici sentivano un doppio obbligo di verificare il contenuto del testo di partenza. Come si è già accennato, un'analisi precisa delle informazioni nuove costituiva un elemento continuo dell'attività delle traduttrici. Questo lavoro portava a volte alla scoperta di alcune imprecisioni che si possono dividere in due categorie: l'obsolescenza dei dati e gli errori di merito. In tale caso ogni traduttrice ha segnalato i frammenti scorretti al redattore scientifico, il che sembra l'unica soluzione possibile, visto che, come afferma Krzysztof Hejwowski, è necessario effettuare le correzioni meritoriche, logiche, stilistiche, perché - contrariamente al caso della traduzione letteraria - "il fatto di non correggere gli errori nei testi informativi è un errore che prima o dopo si vendicherà sul traduttore" 35 .

Infatti, si può supporre che agli occhi del destinatario sia proprio il traduttore che si assume in parte la responsabilità per le informazioni scorrette riportate nel testo. Sarebbe relativamente facile scoprire nel testo tradotto senza correzione dei dati le informazioni che sono diventate obsolete a causa degli avvenimenti politici importanti. Un gran numero degli errori analizzati proviene dalla parte enciclopedica dedicata ai paesi slavi. Per esempio, la Croazia viene descritta come "attualmente candidata all'ingresso nell'Unione Europea" 36 mentre nel 2013 ha avuto luogo l'adesione alla medesima organizzazione. Lo stesso problema viene osservato nelle informazioni sul Montenegro e sulla Serbia che non sono più "candidati potenziali all'ingresso all'Unione europea" ${ }^{37}$, ma candidati ufficiali. Negli ultimi anni è cambiato inoltre il territorio dell'Ucraina che nel momento della pubblicazione della monografia in Italia era ancora "una

35 K. Hejwowski, Kognitywno-komunikacyjna teoria przekładu..., p. 146-147: "Niepoprawianie błędów w tekstach informacyjnych jest błędem, który prędzej czy później zemści się na tłumaczu”.

36 M. Garzaniti, Gli slavi..., p. 27.

37 Ibidem. 
repubblica presidenziale divisa in 24 regioni, una repubblica autonoma (Crimea) e due città a statuto speciale (Kiev e Sebastopoli)"38.

Per di più, fra gli errori di merito si possono elencare i dati scorretti sulla suddivisione della Russia. Lautore ha riportato i dati secondo i quali questo paese è diviso in "47 regioni, 21 repubbliche, 13 territori, due città federali (Mosca e San Pietroburgo)" 39 . Essi non corrispondono tuttavia alle informazioni basate sulla costituzione russa ${ }^{40}$ : attualmente esistono 46 regioni, 22 repubbliche, 9 territori e tre città federali.

Ai polacchi sorprenderebbe probabilmente la costatazione che "il primo letterato slavo a vedersi tributare il premio Nobel fu il polacco Władysław S. Reymont nel 1924", mentre già nel 1905 il premio fu assegnato a Henryk Sienkiewicz ${ }^{41}$. L'affermazione "il re d'Ungheria, Ladislao III Jagellone" potrebbe invece non permetterci di riconoscere in questo personaggio un re polacco, l'erede di Ladislao II di Polonia dalla dinastia dei Jagelloni. Un breve calcolo mostra ancora l'inesattezza dell' informazione che "[q]uando nel 1525 lo stesso ordine [teutonico] si sciolse, la Prussia si trasformò in granducato e il suo gran maestro Alberto di HohenzollernAnsbach, nipote del re di Polonia Sigismondo Augusto, assunse il titolo di granduca, riconoscendosi suo vassallo" ${ }^{\prime 42}$. Il gran maestro fu il nipote di Sigismondo il Vecchio. Il futuro re Sigismondo Augusto ebbe in quel momento cinque anni e nonostante fosse stato incoronato come vivente rege nel 1530 , in realtà avrebbe cominciato a esercitare il potere solo dopo la morte del padre, Sigismondo il Vecchio.

Questo corto elenco di solo alcune imprecisioni trovate nel testo di Marcello Garzaniti attesta ancora una volta il grado di importanza della preparazione adeguata del traduttore. È un aspetto che sembra imprescindibile nel caso della traduzione scientifica. Proprio in questa maniera si potrebbe riassumere probabilmente la parte dell'articolo dedicata alle difficoltà traduttologiche nel lavoro sulla monografia Gli Slavi. Storia, cultura e lingue dalle origini ai nostri giorni di Marcello Garzaniti.

\section{Conclusioni}

La traduzione del testo scientifico - non molto frequente ed estremamente esigente e particolare - è una sfida per il traduttore. Ė un'occasione di studio profondo e arricchente. In questo caso tre traduttrici hanno avuto la possibilità

38 Ibidem, p. 30.

39 Ibidem.

40 http://www.rosjapl.info/historia-rosji-ukrainy-zsrr/podzial-administracyjny-rosji.html (accesso: 4.1.2018).

${ }^{41}$ http://www.noblisci.pl/literacka-nagroda-nobla/page/4/ (accesso: 4.1.2018).

42 M. Garzaniti, Gli slavi..., p. 308. 
di piena cooperazione e sostegno reciproco. Il traduttore nella maggior parte dei casi è esposto soltanto al proprio lavoro, perciò le traduttrici consiglierebbero la cooperazione di almeno due traduttori sui progetti similmente complessi. Nonostante la difficoltà dell'unificazione stilistica, i vantaggi del lavoro condiviso prevalgono e favoriscono la traduzione scientifica. Nella traduzione de Gli slavi. Storia, cultura e lingue dalle origini ai nostri giorni una sfida essenziale si chiudeva nella comprensione del mondo slavo descritto dal punto di vista italiano. Gli altri aspetti lessicali, sintattici e simili sono gli ostacoli minori nei confronti del trasferimento culturale generale del testo. Prendendo in considerazione tutta l'esperienza e la teoria traduttologica si può consigliare una soluzione ovvia e molto importante: di invitare a tradurre le persone specializzatesi nelle discipline richieste, ciò favorirà la velocità di elaborazione e la qualità del testo finale.

Concludendo le nostre riflessioni si sottolinea ancora un aspetto al quale conduce l'analisi del processo traduttivo de Gli Slavi. La pratica della traduzione della monografia di Garzaniti fa capire appieno il ruolo essenziale dello scambio scientifico e culturale tra le nazioni. L'opportunità di comunicare ai lettori polacchi quali idee sulla Slavia dominano tra i rappresentanti di altri paesi è un'esperienza unica che permette di osservare le differenze tra essi. Tuttavia, ancora più visibili sono i fenomeni che ci uniscono. Ci appartengono senza alcun dubbio gli sforzi dei ricercatori e traduttori che nel quadro delle loro attività compiono la missione della diffusione del pensiero e delle informazioni al di là delle differenze linguistiche.

\section{Bibliografia}

Dzierżanowska H., Tłumaczenie tekstów nieliterackich. Założenia teoretyczne i wskazówki metodyczne, Wydawnictwa Uniwersytetu Warszawskiego, Warszawa 1977.

Garzaniti M., Gli slavi. Storia, cultura e lingue dalle origini ai nostri giorni, Carocci, Roma 2013.

Hejwowski K., Kognitywno-komunikacyjna teoria przekładu, Wydawnictwo Naukowe PWN, Warszawa 2004.

Kopczyńska M., Przekład tekstów specjalistycznych - oczekiwania i wyzwania a praktyka, "Rocznik Przekładoznawczy. Studia nad teorią, praktyką i dydaktyką przekładu" 2015, v. 10, p. 113-126, http://dx.doi.org/10.12775/RP.2015.006 (accesso: 29.12.2017).

Kozłowska Z., O przekładzie tekstu naukowego (na materiale tekstów językoznawczych), Wydawnictwa Uniwersytetu Warszawskiego, Warszawa 2007.

Pieńkos J., Podstawy przekładoznawstwa. Od teorii do praktyki, Wydawnictwo Zakamycze, Kraków 2003.

Pisarska A., Tomaszkiewicz T., Wspótczesne tendencje przekładoznawcze, Wydawnictwo Naukowe UAM, Poznań 1996. 
Skibińska E., Miejsce przekładów w „Tekstach” i „Tekstach Drugich”, [in:] Tekst naukowy i jego przekład, a cura di A. Duszak, A. Jopek-Bosiacka, G. Kowalski, Universitas, Kraków 2015.

Wielki słownik poprawnej polszczyzny PWN, a cura di A. Markowski, Wydawnictwo Naukowe PWN, Warszawa 2016.

Wilkoń A., Typologia odmian językowych współczesnej polszczyzny, Wydawnictwo Uniwersytetu Śląskiego, Katowice 2000.

Wojtasiewicz O., Wstęp do teorii tłumaczenia, Wydawnictwo TEPIS, Warszawa 1996.

\section{Sitografia}

www.noblisci.pl/literacka-nagroda-nobla/page/4/ (accesso: 4.1.2018).

www.rosjapl.info/historia-rosji-ukrainy-zsrr/podzial-administracyjny-rosji.html (accesso: 4.1.2018). 